Conclusions Only one study (Mercer 2010) reported a better neurodevelopmental outcome at 7 months of age for preterm infants receiving placental transfusion via DCC. To our knowledge this is the first report on 3.5 year follow-up in infants with DCC or MC. Our results indicate that MC could safely be used as an alternative to DCC. There were no excess events of typical prematurity related co-morbidities in the MC group. Ex-preterm infants seem to benefit from MC and DCC in their neurodevelopmental outcome. Large studies are needed to confirm the findings.

The study is reported on behalf of the Brighton Perinatal Study Group.

\section{PO-0467 MATURATION OF ECHOGENICITY IN PRETERM STRIATUM}

${ }^{1}$ MMA Raets, ${ }^{1} \mathrm{R}$ de Goederen, ${ }^{1} \mathrm{RCJ}$ de Jonge, ${ }^{2} \mathrm{LA}$ Ramenghi, ${ }^{1} \mathrm{KKM}$ Reiss, ${ }^{3} \mathrm{IV}$ Koning, ${ }^{1} \mathrm{P}$ Govaert, ' ${ }^{\prime}$ Dudink. 'Neonatology, Erasmus University Medical Center - Sophia Children's Hospital, Rotterdam, Netherlands; ${ }^{2}$ Neonatology, Istituto Giannina Gaslini, Genoa, Italy; ${ }^{3}$ Obstetrics, Erasmus University Medical Center - Sophia Children's Hospital, Rotterdam, Netherlands

\subsection{6/archdischild-2014-307384.1108}

Background and aims Preterm infants are at risk of brain injury. Cranial ultrasound is frequently used in neonatal care to detect and monitor brain injury. Anatomical structures and abnormalities can be distinguished by differences in echogenicity.

Our primary objective was to reliably measure sonographic grey values in basal ganglia. Secondary objectives included the influence of gestational age at birth on echogenicity and aspects of deep grey matter change at 30 weeks corrected GA.

Methods We prospectively collected CUS-data of 229 preterm infants ( $<29$ weeks gestation). Parasagittal images through the gangliothalamic ovoid were assessed on mean grey value in putamen and globus pallidus. Intra- and interobserver for placement of ROI were analysed.

Results The method used produced a reliable globus pallidus to putamen ratio (GPP ratio). Mean GPP ratio was $0.786( \pm 0.085)$. Extreme preterm infants have significantly lower GPP at birth than did preterm infants above 28 weeks $(0.755 \pm 0.081$ vs 0.808 \pm 0.091 ; P-value $<0.01)$. At 30 weeks corrected GA this was still the case $(0.723 \pm 0.051$ vs. $0.818 \pm 0.063$; P-value $<0.01)$.

Conclusion The putamen of extremely preterm infants is more hyperechoic then putamen of preterm infants of 29 weeks of gestation. Objective measurement of grey values can help to study brain injury.

\section{PO-0468 ERYTHROPOIETIN FOR THE REPAIR OF CEREBRAL INJURY IN VERY PRETERM INFANTS (EPOREPAIR) - A RANDOMISED, DOUBLEBLIND AND MULTICENTRE INTERVENTIONAL STUDY}

${ }^{1} \mathrm{CM}$ Rüegger, ${ }^{1} \mathrm{C}$ Hagmann, ${ }^{1} \mathrm{~B}$ Koller, ${ }^{2} \mathrm{C}$ Bührer, ${ }^{1} \mathrm{HU}$ Bucher, ${ }^{3} \mathrm{~S}$ Wellmann. ${ }^{1}$ Division of Neonatology, University Hospital Zurich, Zurich, Switzerland; '2Department of Neonatology, Charité University Medical Centre, Berlin, Germany; ${ }^{3}$ Division of Neonatology, University Children's Hospital, Basel, Switzerland

\subsection{6/archdischild-2014-307384.1109}

Background Preterm infants suffering from intraventricular haemorrhage (IVH) are at increased risk for neurodevelopmental impairment. Observational data suggest that recombinant human erythropoietin (EPO) aimed at preventing anaemia also improves long-term cognitive outcome in infants with IVH (Neubauer AP et al., Annals Neurology, 2010). The recently completed first early high-dose EPO trial in very preterm infants did not raise any significant safety concerns (Fauchère J-C. et al., ESPR Annual Meeting, 2012). Hypothesis: High-dose EPO improves long-term neurodevelopmental outcome in preterm infants with IVH.

Methods Design: Double blind, 1:1 randomised clinical study in 11 perinatal centres (Germany and Switzerland). Patients: 120 very preterm (gestational age $<32$ weeks) and/or very low birth weight $(<1500 \mathrm{~g})$ infants with IVH $\left(>\mathrm{I}^{\circ}\right)$ diagnosed by cranial ultrasound during the first 4 days of life. Intervention: 5 intravenous applications of EPO $(2000 \mathrm{U} / \mathrm{kg})$ or placebo spread over 3 weeks. Primary objective: Neurodevelopmental outcome at 5 years of age (Kaufmann-ABC or Son-R). Secondary objectives: (1) safety; (2) MRI at term equivalent age to quantitatively analyse brain injury and growth; (3) psychomotor development at 2 years of age (BSID-III). Recruitment: March 2014 to February 2016.

Results and conclusions Given the fact that long-term neurodevelopmental outcome cannot be reliably assessed until preschool age, the primary outcome of this study providing evidence as to whether high-dose EPO improves restitution of brain damage in preterm infants will not be reported before 2021. However, MRI data can be reported much earlier. (Funded by the Swiss National Science Foundation; Clinical Trials Registry: NCT02076373).

\section{P0-0469 ALLOMETRIC SCALING OF BRAIN GROWTH IN PRETERM INFANTS AND IN PIGLETS}

${ }^{1} \mathrm{AM}$ Plomgaard, ${ }^{2} \mathrm{AD}$ Andersen, ${ }^{2} \mathrm{~T}$ Thymann, ${ }^{2} \mathrm{PT}$ Sangild, ${ }^{1} \mathrm{G}$ Greisen. ${ }^{1}$ Department of Neonatology, Copenhagen University Hospital Rigshospitalet, Copenhagen, Denmark; ${ }^{2}$ Department of Nutrition Exercise and Sports Faculty of Science, University of Copenhagen, Frederiksberg, Denmark

\subsection{6/archdischild-2014-307384.1110}

Background The relationship between brain size (measured as head circumference) and body weight in human infants is allometric. This means that the relative growth rates of the body and the brain stays in a constant relation during infancy. We are in the process of developing a preterm piglet model to study nutritional interventions on the brain. Here we present an analysis of the growth pattern during the first weeks of life.

Materials and methods Piglets $(\mathrm{n}=146)$ were delivered by planned C-section at $90 \%$ and $100 \%$ gestation. All piglets were part of nutritional intervention studies in which daily body weight gain and body and brain weight upon euthanasia (d0-26) were obtained.

Results An allometric scaling model was established by linear regression using the log-transformed values of brain and body weight for piglets at 4 different ages at euthanasia: -10d (preterm at birth), $0 \mathrm{~d}$ (term at birth), $5 \mathrm{~d}$ and $26 \mathrm{~d}$ for term piglets. Preterm piglets aged 4-26 days at euthanasia $(n=52)$ gained less weight after birth compared to term (12 vs. $26 \mathrm{~g} /(\mathrm{kg} * \mathrm{~d}),(\mathrm{p}<$ 0.01 ), but the relation between body and brain weight did not deviate from the allometric scaling model (mean Z-score 0.014, $\mathrm{p}=0.94)$. 